\title{
Prevenção da violência doméstica: Importância da intervenção do enfermeiro gestor de caso
}

\section{Ana Paula Reis Carmona (iD}

Agrupamento de Centros de Saúde da Amadora - Lisboa - Portugal

Maria da Graça Silva Quaresma iD

Centro Hospitalar e Universitário de Lisboa Central - Lisboa - Portugal

Pedro Ricardo Martins Bernardes Lucas (DD

Escola Superior de Enfermagem de Lisboa - Lisboa - Portugal

\section{RESUMO}

Objetivos: Identificar evidência científica sobre intervenção do enfermeiro gestor de caso, na prevenção da violência doméstica. Métodos: Revisão Integrativa da Literatura de estudos indexados na plataforma EBSCOHost (LILACS, SCOPUS e Web of Science) e bases de dados CINAHL, MEDLINE (PubMed/Medline) e Cochrane. Os estudos selecionados são alusivos ao período de 10 anos (2010 a 2020); Resultados: Foram selecionados 11 artigos sobre implementação de programas de deteção e prevenção da violência doméstica com resultados para as vítimas, os enfermeiros, os enfermeiros gestores e na eficiência das organizações, tanto em Atenção Primária em Saúde como em Hospitais. Conclusão: A implementação de modelos de gestão de caso promove melhor resposta às vítimas de violência, com implicações significativas na qualidade dos cuidados e no aumento de resultados na prevenção. Não foram encontrados estudos referentes à Atenção Primária em Saúde referindo a importância do enfermeiro gestor de caso na prevenção da violência doméstica.

Descritores: Atenção Primária em Saúde; Violência Doméstica; Enfermagem; Administração de Caso; Revisão.

\section{INTRODUÇÃO}

A violência doméstica (VD) é uma problemática transversal a toda a sociedade, tendo um impacto expressivo na qualidade de vida dos indivíduos e na saúde das famílias e comunidades, com consequente aumento dos gastos em saúde. É um fenómeno multifatorial, definido pela Organização Mundial de Saúde como; o uso de força física ou poder contra si mesmo, ou contra outra pessoa, grupo ou comunidade e que tem como consequência a possibilidade de aparecimento de lesões físicas, danos psicológicos, alterações de desenvolvimento, abandono ou negligência, e inclusivamente a morte ${ }^{(1)}$.

A Organização para a Cooperação e Desenvolvimento Económico afirma que a principal finalidade de um sistema de saúde é a promoção da saúde dos usuários e das populações. Ser um indivíduo saudável implica o desenvolvimento do bem-estar pessoal, psicológico e socioeconómico, pessoas saudáveis geram comunidades saudáveis e incrementam uma sociedade ativa, mais produtiva e próspera. Uma prática profissional de atendimento positiva é um sinal importante para a prestação de cuidados de qualidade(2).

Perante esta preocupação e em virtude de existir um escasso conhecimento sobre a evidência científica relacionada com a intervenção do enfermeiro gestor de caso em contexto de Atenção Primária em Saúde (APS), em situações de violência doméstica, é indispensável e uma obrigação, desenvolver a investigação em enfermagem sobre a temática. Derivado principalmente do fato de os estudos publicados serem escassos e pouco divulgados, ocorreu assim a oportunidade de investigar a intervenção do enfermeiro gestor de caso para além do contexto hospitalar, em outros níveis de prestação de cuidados no sistema de saúde.

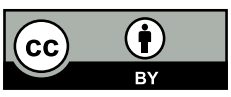

Este artigo está publicado em acesso aberto (Open Access) sob a licença Creative Commons, que permite uso, distribuição e reprodução em qualquer meio, sem restrições, desde que o trabalho seja corretamente citado. 
Identificando o gestor de caso como um coordenador de intervenção de cuidados integrados e complexos, detendo uma contribuição única para os cuidados sociais e de saúde, assim como na participação e na autonomia dos indivíduos em situações complexas de saúde ${ }^{(3)}$, torna-se pertinente a compreensão, da influência da intervenção do enfermeiro gestor de caso, ou do gestor clínico, em situação de violência doméstica, em Atenção Primária à Saúde. Pretende-se assim responder à questão de revisão: Qual a evidência científica sobre a intervenção do enfermeiro gestor de caso, na promoção da prevenção da violência doméstica?

Este estudo pretende dar a conhecer a evidência científica sobre esta temática, através da utilização do método de revisão com base nos pressupostos da revisão integrativa da literatura, com Prática Baseada na Evidência (PBE). Foram incluídos, apesar de não serem contemplados na técnica da revisão integrativa da literatura, artigos de revisão, dissertações e de opinião, pela sua pertinência e escassez de artigos com evidência científica, sobre a temática específica escolhida. Para tal foi delineado como objetivo: Identificar a evidência científica sobre a importância da intervenção do enfermeiro gestor de caso, na prevenção da violência doméstica(4)

\section{MÉTODOS}

Esta revisão teve como referência a metodologia proposta por Whittemore e Knafl(5) para as revisões integrativas da literatura. O objetivo delineado foi "Identificar a evidência científica sobre a intervenção do enfermeiro gestor de caso, na prevenção da violência doméstica", sendo a questão de revisão: Qual a evidência científica, sobre a intervenção do enfermeiro gestor de caso na promoção da prevenção da violência doméstica ${ }^{(4)}$

Foi definido um limite temporal alargado de 10 anos para que fosse possível abranger toda a literatura que abordasse a temática em questão, devido à escassez da temática na APS, no contexto do papel do Enfermeiro gestor de caso em situação de violência doméstica. Assim, os artigos obtidos foram então limitados ao período de 2010 a 2020. Esta revisão examinou os estudos publicados em português, inglês e espanhol.

Foi escolhida a plataforma EBSCOhost e selecionaram-se as bases bibliográficas eletrónicas: CINAHL, MEDLINE e Cochrane Database.

Foram considerados os descritores validados através dos Descritores em Ciências da Saúde - DeCS: Violência Doméstica (Domestic Violence), Atenção Primária em Saúde (Primary Health Care), Administração de Caso (Case Management), Saúde Pública (Public Health), Enfermagem (Nursing). Estes foram combinados através das expressões booleanas AND da seguinte forma: Domestic Violence AND Primary Health Care AND Nursing numa primeira pesquisa, tendon uma segunda pesquisa sido combinados utilizando Domestic Violence AND Case Management AND Nursing.

Foram considerados assim os estudos que continham os critérios de elegibilidade, ou seja, os artigos disponíveis em texto completo, os artigos indexados nos últimos 10 anos, que enquadrassem a temática da prevenção da violência doméstica, na APS, mas também o papel de enfermeiro gestor de caso, de igual forma enquadrado na temática da violência doméstica( ${ }^{(4)}$.

Após terem sido eliminados os artigos em duplicado, foram identificados 142 artigos para a seleção da revisão. A seleção dos estudos foi realizada após a leitura atenta dos títulos dos 142 artigos, tendo ficado excluídos desta forma 52 artigos. Pela leitura minuciosa dos resumos foram perscrutados 90 artigos, sendo excluídos mais 72 artigos. Desta forma, foram apenas considerados os estudos que estavam de acordo com os critérios de inclusão supracitados, tendo sido examinados integralmente 18 artigos, aos quais foram acrescentados 5 pela sua pertinência para esta revisão e por cumprirem os critérios de inclusão anteriormente delineados, tendo assim sido examinados integralmente 23 artigos. Foram finalmente selecionados para este estudo $11 \operatorname{artigos}^{(4)}$.

Na Figura 1 estão explanados os resultados das etapas de análise, segundo o modelo PRISMA Flow Diagram ${ }^{(6)}$.

À posteriori, foi realizada a leitura integral e aprofundada dos 11 artigos selecionados. Os dados obtidos foram extraídos dos artigos incluídos na revisão, através da utilização de uma tabela de extração dos resultados, de acordo com o objetivo e a pergunta da revisão. A tabela de extração foi organizada da seguinte forma: Autor(es), Ano de publicação, País; Título; Objetivos; Desenho do estudo; População em estudo/Tamanho da amostra/Participantes; Contexto; Conceito(s) relevantes da questão de revisão; Principais conclusões. Objetivo da revisão, resultados encontrados e implicação para a gestão em enfermagem. 


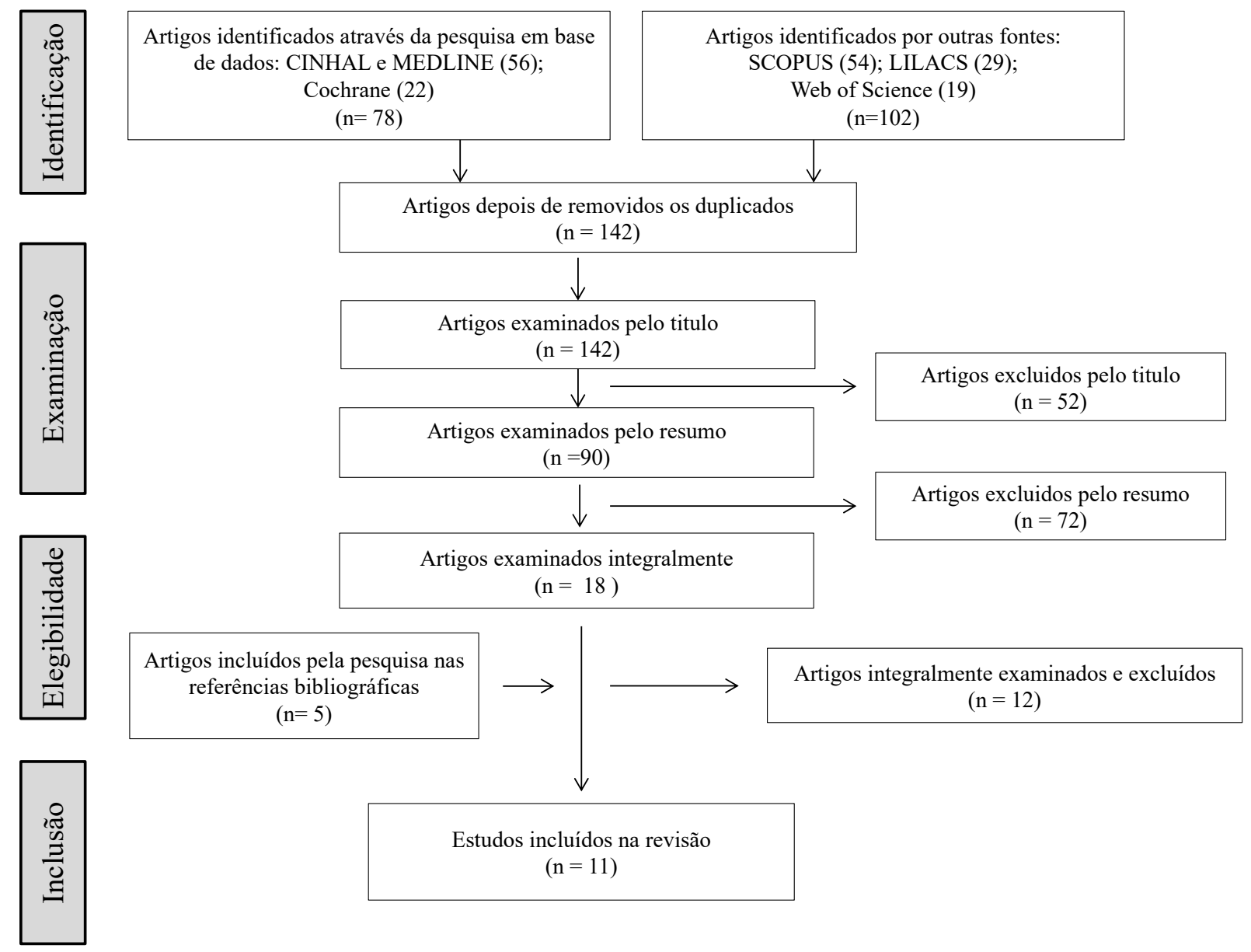

Figura 1 - Diagrama PRISMA referente ao processo de seleção dos estudos ${ }^{(4)}$.

\section{RESULTADOS}

Uma vez obtidos os artigos de texto integral, foram lidos e estudados minuciosamente, de modo a obter a amostra final de 11 artigos que cumpriam os critérios de inclusão, dos quais 4 exclusivamente em contexto da APS, 1 em contexto hospitalar e 6 em contexto misto, ou seja, todos os contextos de prestação de cuidados; APS, hospitais, e organizações não-governamentais.

O artigo mais recente foi publicado no ano de 2019 e o mais antigo no ano de 2012, sendo um de 2013, um de 2014, três de 2015, dois de 2016 e dois de 2017. Os Estados Unidos da América estão representados com o maior número de publicações, que foram quatro. O Brasil com uma publicação, enquanto a Europa apresentou também quatro publicações, sendo três do Reino Unido e uma de Espanha. Dois dos estudos selecionados são ainda de origem Australiana.

Foram também encontradas duas revisões sistemáticas da literatura publicadas em 2016 e 2017, tendo uma direcionado o seu estudo para a temática do gestor de caso e sua contribuição para a integração de cuidados aplicados à APS, enquanto que a outra investigou os variados programas existentes em curso, de assistência em saúde para vítimas de violência doméstica, respetivamente.

Embora tenha sido importante incluir revisões sistemáticas, apenas duas delas incidiam no contexto de cuidados definido para esta revisão, que são metassínteses.

Uma reavisão(7) ${ }^{(7)}$ analisou um total de 43 estudos que consideraram o fenómeno da violência doméstica como complexo e um importante problema de Saúde Pública, explicando a multiplicidade e heterogeneidade do mesmo quanto ao tipo de assistência, como ela é prestada, ou como os próprios estudos são avaliados, o que nos cria dúvidas e desafios sobre a perceção de quais são realmente eficazes ${ }^{(7)}$. Outra revisão(3) que analisam os fatores relacionados com a gestão de casos, com todas as suas diferentes denominações, variações e em diversos 
contextos, continuando a sustentar a coordenação, a integração e a gestão dos cuidados e assistência social, em muitos contextos diferentes, para diferentes condições de saúde, também em contexto de APS ${ }^{(3)}$.

No Quadro 1 apresenta-se um quadro síntese da extração dos artigos incluídos na presente revisão.

Quadro 1 - Síntese da extração dos artigos incluídos na revisão.

\begin{tabular}{|c|c|c|c|c|}
\hline Autores & Ano & Pais & Titulo do Artigo/Periódico & Tipo de Estudo \\
\hline Muller, L. S. & 2014 & $\begin{array}{l}\text { Estados } \\
\text { Unidos }\end{array}$ & $\begin{array}{l}\text { "A Case Management Briefing on Domestic } \\
\text { Violence" - Professional Case Management }\end{array}$ & Artigo de opinião \\
\hline $\begin{array}{l}\text { Silva, N. N. F., Leal, S. M. C., } \\
\text { Trentin, D., Vargas, M. A. D. O., } \\
\text { Vargas, C. P., \& Vieira, L. B. }\end{array}$ & 2017 & Brasil & $\begin{array}{l}\text { "Atuação dos enfermeiros da atenção básica } \\
\text { a mulheres em situação de violência" - } \\
\text { Enfermagem em Foco }\end{array}$ & $\begin{array}{l}\text { Estudo qualitativo, } \\
\text { descritivo }\end{array}$ \\
\hline Hooker, L., \& Taft, A. & 2016 & Australia & $\begin{array}{l}\text { "Review: Using theory to design, implement } \\
\text { and evaluate sustained nurse domestic } \\
\text { violence screening and supportive care" - } \\
\text { Journal of Research in Nursing }\end{array}$ & Dissertação \\
\hline $\begin{array}{l}\text { Sprague, S., Scott, T., } \\
\text { Garibaldi, A., Bzovsky, S., } \\
\text { Slobogean, G. P., McKay, P., } \\
\text { Spurr, H., Arseneau, E., } \\
\text { Memon, M., Bhandari, M., \& } \\
\text { Swaminathan, A. }\end{array}$ & 2017 & $\begin{array}{l}\text { Reino } \\
\text { Unido }\end{array}$ & $\begin{array}{l}\text { "A scoping review of intimate partner violence } \\
\text { assistance programmes within health care } \\
\text { settings" - European Journal of } \\
\text { Psychotraumatology }\end{array}$ & Revisão Scoping \\
\hline $\begin{array}{l}\text { Kramer, A., Nosbusch, J. M., \& } \\
\text { Rice, J. }\end{array}$ & 2012 & $\begin{array}{l}\text { Estados } \\
\text { Unidos }\end{array}$ & $\begin{array}{l}\text { "Safe Mom, Safe Baby -A Collaborative } \\
\text { Model of Care for Pregnant Women } \\
\text { Experiencing Intimate Partner Violence" - } \\
\text { Journal of Perinatal \& Neonatal Nursing }\end{array}$ & $\begin{array}{l}\text { Projecto Inovador; } \\
\text { Programa } \\
\text { Interdisciplinar }\end{array}$ \\
\hline Basu, S., \& Ratcliffe, G. & 2013 & $\begin{array}{l}\text { Reino } \\
\text { Unido }\end{array}$ & $\begin{array}{l}\text { "Developing a multidisciplinary approach } \\
\text { within the ED towards domestic violence } \\
\text { presentation" - Emergency Medicine Journal }\end{array}$ & $\begin{array}{l}\text { Projecto Quality } \\
\text { Improvement }\end{array}$ \\
\hline $\begin{array}{l}\text { Lukersmith, S., Millington, M., \& } \\
\text { Salvador-Carulla, L. }\end{array}$ & 2016 & Australia & $\begin{array}{l}\text { "What is Case Management? A Scoping and } \\
\text { Mapping Review" - International Journal of } \\
\text { Integrated Care }\end{array}$ & Revisão Scoping \\
\hline $\begin{array}{l}\text { Prieto S, G., Buendía E, L., } \\
\text { Corbalán M C, M., \& Mogente } \\
\text { A, C. }\end{array}$ & 2015 & Espanha & $\begin{array}{l}\text { "Cribado de violencia de género em un } \\
\text { departamento de salud" - Revista } \\
\text { Investigacion \& Cuidados }\end{array}$ & $\begin{array}{l}\text { Análise observacional } \\
\text { transversal } \\
\text { retrospectiva. }\end{array}$ \\
\hline Armold, S. & 2019 & $\begin{array}{l}\text { Estados } \\
\text { Unidos }\end{array}$ & $\begin{array}{l}\text { "Case management: An overview for nurses" } \\
\text { - Nursing }\end{array}$ & Artigo de opinião \\
\hline Bradbury-Jones, C. & 2015 & $\begin{array}{l}\text { Reino } \\
\text { Unido }\end{array}$ & $\begin{array}{l}\text { "Talking about domestic abuse: Crucial } \\
\text { conversations for health visitors" - Community } \\
\text { Practitioner }\end{array}$ & $\begin{array}{l}\text { Estudo qualitativo, } \\
\text { descritivo }\end{array}$ \\
\hline Davis, $\mathrm{K}$. & 2015 & $\begin{array}{l}\text { Estados } \\
\text { Unidos }\end{array}$ & $\begin{array}{l}\text { "Innovative Models of Care Delivery: } \\
\text { Addressing Transitions Across The Care } \\
\text { Continuum" - Health Forum/AHA Leadership } \\
\text { Summit }\end{array}$ & $\begin{array}{l}\text { Projecto Inovador; } \\
\text { Programa } \\
\text { Interdisciplinar }\end{array}$ \\
\hline
\end{tabular}

\section{DISCUSSÃO}

A criatividade e análise crítica da exibição dos dados é fundamental para a comparação dos mesmos assim como para a identificação de temas e padrões importantes ${ }^{(5)}$; foram então usados alguns dos elementos da análise de dados descritos pelos autores: Foram observados os padrões e os temas dos artigos encontrados, vendo plausibilidade, 
seguidamente foram construídos contrastes e comparações, discernindo padrões comuns e incomuns, incluindo detalhes em geral, observando as relações e encontrando os fatores intervenientes.

Pela análise dos resultados obtidos nos 11 artigos, foram construídas as cinco categorias lógicas temáticas de evidência: O gestor de caso em situação de VD e a responsabilidade multifacetada; a contribuição do gestor de caso para situações de saúde complexas; a complexa abordagem em contexto de APS da temática da VD; modelos de abordagem do fenómeno da VD para a prevenção da mesma e diminuição de complicações; e ainda programas e projetos inovadores, com equipas interdisciplinares para a prestação de cuidados de qualidade a usuários com situações de saúde complexas ${ }^{(4)}$.

\section{O gestor de caso em situação de violência doméstica e a responsabilidade multifacetada}

O gestor de caso detém responsabilidade na promoção da prevenção da violência doméstica. Para além de se manter atualizado em relação às questões que afetam a sua prática profissional, também deve ter sempre presente os seus deveres jurídicos e profissionais. O gestor de caso tem um papel primordial, pois pode fazer a diferença ao ponto de salvar uma vida, pelo acompanhamento ou encaminhamento das situações, através dos registos e da notificação das situações de risco/perigo por ele avaliadas ${ }^{(8)}$, tendo ainda um importante papel na capacitação da comunidade para implementar estratégias de forma a quebrar o ciclo da perpetração da $\mathrm{VD}^{(9)}$.

Os autores enfatizam ainda nos seus artigos a importância da existência de um profissional gestor de caso para o fenómeno da VD. Este profissional deve possuir conhecimentos desenvolvidos e atualizados sobre a temática, por forma a trabalhar tanto com os profissionais atualizando-os e colaborando na resolução das situações, com os usuários e as próprias comunidades na sensibilização, acompanhamento e sempre que necessário com formação, para a prevenção da VD.

\section{A contribuição do Gestor de caso para situações de saúde complexas}

A gestão de casos proporciona uma contribuição única para a integração em cuidados de saúde, serviços sociais e outros serviços no setor da APS para as pessoas com condições de saúde complexas ${ }^{(3)}$. Também os enfermeiros prestando cuidados como gestores de caso, "advogam" os usuários, contribuindo com as suas atividades e competências, também para a diminuição de custos, bem como para outros benefícios que ajudam a suportar os sistemas de saúde ${ }^{(10)}$.

Nestes artigos encontram-se bem elucidada e desenvolvida a aceção de competências de Gestor de caso para resolução de situações de saúde complexas, qual a importância e as características das suas atividades e funções na APS, enfatizando as mais valias em termos da qualidade dos cuidados prestados tal como dos benefícios económicos, para os sistemas de saúde. Defendem ainda a importância da implementação do gestor de caso clínico, mais especificamente do enfermeiro, no enquadramento das suas atividades e funções profissionais.

\section{A complexa abordagem em contexto da APS da temática da VD}

A violência doméstica é considerada como um grave problema de saúde pública, sendo que as enfermeiras desempenham um papel importante no reconhecimento e na resposta ao fenómeno ${ }^{(11)}$. Muitos profissionais possuem conhecimentos sobre violência doméstica, mas não sentem segurança suficiente para questionar os usuários, clientes e alunos/colegas, por forma a identificar as situações ${ }^{(11)}$. Os profissionais que realizam as visitas domiciliárias, quer sejam enfermeiros, fisioterapeutas, ou outros, que trabalham com e para a comunidade, têm aí uma janela de oportunidade e desempenham um papel importante no início de conversas determinantes sobre assuntos domésticos e de violência doméstica, podendo também utilizar as suas competências relacionais para melhorar os resultados de saúde pública tanto para as crianças, como para as famílias ${ }^{(11)}$.

Neste artigo o autor desenvolve a importância da prevenção da VD na APS. Investir na formação e desenvolvimento da sensibilização dos enfermeiros da APS é fundamental para prevenir a VD. São estes profissionais que estão disponíveis aos usuários e seus familiares, dentro das organizações de saúde, nas visitas domiciliárias e nas atividades várias com a comunidade.

\section{Modelos de abordagem do fenómeno da VD para a prevenção e diminuição de complicações}

Um estudo ${ }^{(12)}$ utilizou variados instrumentos de medida e avaliação da VD entre os quais o questionário "Abuse Assessment Screen" (AAS). Este questionário é usado como um instrumento de triagem para avaliação de situações de risco/perigo de violência doméstica. Em caso de confirmação de um caso positivo de VD, existe a obrigação legal 
de completar o registo e a documentação com uma parte específica das lesões. Outra forma de realizar uma triagem do fenómeno da VD por forma a analisar o risco é também, usando o questionário "Danger Assessment" (DA) ${ }^{(12)}$.

O programa MOVE desenvolvido por um estudo(13) "Improving maternal and child health care for vulnerable mothers", foi um ensaio clínico randomizado realizado a partir de um modelo aperfeiçoado de triagem da violência doméstica e respetivos cuidados de suporte, prestados por parte de enfermeiros de saúde materna e infantil. Foi desenvolvido a partir do Modelo de Processo de Normalização (NPT), uma teoria de implementação social, que fornece uma construção conceptual para compreender o planeamento social do trabalho (implementação), de transformar os seus procedimentos como elementos usuais na vida quotidiana (incorporação) e de manter práticas integradas nos seus contextos sociais (integração). O "Normalization Process Theory" (NPT) desenvolveu a introdução de uma nova prática clínica como um processo social ${ }^{(14)}$

Foi exposto ainda outro programa ${ }^{(15)}$ "Safe mom, safe baby" (SMSB), um programa clínico interdisciplinar, liderado por enfermeiras, que conquistou ganhos no tratamento do complexo problema da VD, durante a gravidez e nas suas consequências. Estes ganhos refletem o impacto do modelo de prestação colaborativa de cuidados, identificando especificamente a forma como o modelo sustenta a integração clínica, promovendo sinergias entre os recursos por vezes escassos e conjuntos de competências, do sistema de saúde e da "agência comunitária" de violência doméstica(15). Os resultados positivos relacionados com saúde e segurança para as mulheres e seus bebés são atingidos, tal como os profissionais das equipas das organizações de saúde e violência doméstica beneficiam dos serviços de consultoria e educacionais do programa ${ }^{(15)}$.

Foi ainda encontrada a descrição de um outro projeto de melhoria de qualidade sobre a criação, implementação e avaliação de um programa estruturado para detetar e gerir situações de VD, num serviço especializado em medicina de emergência ${ }^{(16)}$. Foi reconhecido que uma forte liderança, tal como a priorização do tema dentro do serviço, facilita o desenvolvimento dos procedimentos e contribui substancialmente para o seu sucesso. $O$ trabalho em rede e parceria, realizado com os parceiros da comunidade, é inestimável na adaptação do programa de prestação de serviço e educação, às necessidades dos funcionários e usuários ${ }^{(16)}$.

Estes autores concluíram ser possível prevenir a VD, tal como diminuir as complicações que dela advêm, através da implementação dos seus programas em cuidados hospitalares ${ }^{(16)}$. Demonstraram ser essencial a triagem, sistematizada, para avaliação de situações de risco/perigo de violência doméstica, o registo adequado, assim como o trabalho em rede, com os parceiros comunitários e as próprias famílias ${ }^{(16)}$.

\section{Programas e projetos inovadores, com equipas interdisciplinares para a prestação de cuidados de qualidade,} a usuários com situações de saúde complexas

A maioria dos diferentes tipos de programas de assistência a mulheres em situações de saúde complexas, vítimas de violência doméstica, apresentam resultados positivos e foram considerados como benéficos para as mesmas ${ }^{(7)}$.

Um dos projetos inovadores implementado é descrito como tendo equipas de médicos e enfermeiros que realizam visitas aos usuários, prestam cuidados de saúde no domicílio, sendo que as equipas também incluem assistentes sociais, fisioterapeutas ou até capelães ${ }^{(17)}$. A instalação de modelos de atendimento inovadores, envolve todo um sistema e não unicamente uma linha de serviço, ou uma especialidade ${ }^{(7)}$. A instalação dos mesmos requer

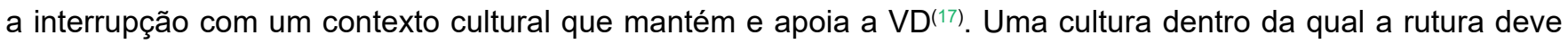
ocorrer é aquela construída sobre alinhamento, visão compartilhada, propósito compartilhado, comunicação, fatores protetores identificados e a continuidade do cuidado ${ }^{(17)}$.

Nestes artigos foi demonstrada a importância da prestação de cuidados de qualidade, aos usuários em situação de saúde complexa, como sendo a situação das vítimas de VD. Apresentam-nos projetos e programas inovadores, realizados por equipas multidisciplinares orientadas pelo gestor de caso, com interligação em rede de parceria, de resposta eficaz e adequada às situações em que as usuárias se encontram.

Devido à especificidade do tema do enfermeiro gestor de caso e do contexto da prevenção da violência doméstica na APS, bem como à respetiva escassez de evidência científica, uma limitação deste estudo foi a existência de apenas três artigos nos últimos três anos do período de pesquisa. Em relação aos últimos cinco anos do período temporal da pesquisa, os estudos incluídos foram apenas cinco, do total dos onze analisados nesta revisão. Outra das possíveis limitações desta revisão prende-se com o fato de apenas estarem incluídos estudos publicados em inglês, português e espanhol. Artigos publicados em outros idiomas também poderiam ter sido importantes para esta revisão.

Apesar da existência de um reduzido conhecimento sobre o enfermeiro gestor de caso para a prevenção da violência doméstica na APS, esta revisão de literatura pretende contribuir para sistematizar esse conhecimento nas 
diferentes áreas de atuação dos enfermeiros: na prestação de cuidados, na gestão, na investigação, na docência e na consultoria ou assessoria.

\section{CONCLUSÃO}

Nos artigos com critérios de elegibilidade desta revisão, foi encontrada alguma evidência científica sobre a mais valia do enfermeiro gestor de caso para a situação de VD, em contexto da APS. Fornece-nos assim alguns contributos para a melhoria da qualidade dos cuidados de saúde e particularmente de enfermagem, a pessoas em situação de VD, em contexto da APS, prestando um serviço aos enfermeiros, aos enfermeiros gestores, aos académicos, aos investigadores e ainda aos responsáveis pela definição de políticas de saúde.

Enfatiza-se, ainda, a importância de aspetos a considerar em contexto da APS, como a relevância de uma relação multidisciplinar de qualidade entre enfermeiros e médicos, assim como entre enfermeiros, gestores e gestores. Também demonstra a significativa consideração de uma boa comunicação nas Unidades de Saúde e entre as organizações, por forma a realizar um trabalho em rede, de prevenção do fenómeno da violência doméstica. Os vários estudos encontrados, demonstram através da implementação de programas de melhoria da qualidade de cuidados no âmbito da VD, que a intervenção do enfermeiro gestor de caso, enquanto líder, em equipas multiprofissionais, melhora a comunicação em rede, com os profissionais e usuários, conduzindo a benefícios económicos e ganhos em saúde, demonstrando ainda a satisfação dos profissionais neles implicados.

O fenómeno de VD como complexo que é, deve ser compreendido e trabalhado em contexto da APS, em interligação com os parceiros comunitários, mas também em contexto hospitalar, pelo tipo de tomada de decisão, relação entre os membros das equipas e pelos próprios processos organizacionais. Também é necessário promover e implementar, tal como defendido em alguns dos estudos, a metodização e o rastreio/triagem das situações de VD, o que vai contribuir para a prevenção da mesma assim como para o aumento dos resultados com os clientes, enfermeiros e organizações, ou seja, com todos os atores envolvidos.

Vários estudos demonstram ainda que para atingir essa finalidade se apresenta como mais-valia o enfermeiro gestor de caso que, pelas suas competências e características profissionais, pode contribuir também para a redução de custos, entre outros benefícios, que ajudam a sustentar os sistemas de saúde.

\section{AGRADECIMENTOS E CONFLITOS DE INTERESSE}

Agradecemos ao CIAIQ 2021 a oportunidade de realizar a divulgação científica respeitante a esta temática. Os autores declaram não existir qualquer conflito de interesses.

\section{CONTRIBUIÇÕES}

Todos os autores contribuíram com a elaboração e o delineamento do estudo; a aquisição, a análise e a interpretação de dados; e a redação e/ou a revisão do manuscrito e todos aprovaram a versão final enviada para publicação na Revista Brasileira em Promoção da Saúde (RBPS) e são responsáveis por todos aspectos do trabalho.

\section{REFERÊNCIAS}

1. Taft AJ, Small R, Humphreys C, Hegarty K, Walter R, Adams C, et al. Enhanced maternal and child health nurse care for women experiencing intimate partner/family violence: protocol for MOVE, a cluster randomised trial of screening and referral in primary health care. BMC Public Health. 2012; 12:811.

2. OECD - Organisation for Economic Co-operation and Development. Health at a Glance 2019 - OECD indicators. Paris: OECD Publishing; 2019. (Health at a Glance).

3. Lukersmith S, Millington M, Salvador-Carulla L. What is case management? A scoping and mapping review. Int J Integr Care. 2016;16(4):1-13.

4. Carmona A, Quaresma G, Lucas P. Enfermeiro Gestor de Caso na promoção da prevenção da Violência Doméstica - Revisão Integrativa. New Trends Qual Res. 2021;8:106-13.

5. Whittemore R, Knafl K. The integrative review: updated methodology. Methodol Issues Nurs Res. 2005;52(5):546-53. 
6. Moher D, Liberati A, Tetzlaff J, Altman D. Preferred Reportinf Items for Systematic Reviwes and MetaAnalyses: The PRISMA Statment. PLoS Med. 2009;6:1-2.

7. Sprague S, Scott T, Garibaldi A, Bzovsky S, Slobogean GP, McKay P, et al. A scoping review of intimate partner violence assistance programmes within health care settings. Eur J Psychotraumatol. 2017;8(1):13.

8. Silva NNF, Leal SMC, Trentin D, Vargas MADO, Vargas CP, Vieira LB. Atuação dos enfermeiros da atenção básica a mulheres em situação de violência. Enferm em Foco. 2017;8(3):70-4.

9. Muller LS. A case management briefing on domestic violence. Prof Case Manag. 2014;19(5):237-40.

10. Armold S. Case management: An overview for nurses. Nursing (Lond). 2019;49(9):43-5.

11. Bradbury-Jones $C$. Talking about domestic abuse: Crucial conversations for health visitors. Community Pract. 2015;88(12):40-3.

12. Prieto SG, Buendía EL, Corbalán MCM, Mogente AC. Cribado de violencia de género en un departamento de salud. Rev Investig Cuid. 2015;13(33):6-12.

13. Taft AJ, Hooker L, Humphreys C, Hegarty K, Walter R, Adams C, et al. Maternal and child health nurse screening and care for mothers experiencing domestic violence (MOVE): A cluster randomised trial. BMC Med. 2015;13(1):1-10.

14. Hooker L, Taft A. Using theory to design, implement and evaluate sustained nurse domestic violence screening and supportive care. J Res Nurs. 2016 Sep;21(5/6):432-42.

15. Kramer A, Nosbusch JM, Rice J. Safe mom, safe baby: A collaborative model of care for pregnant women experiencing intimate partner violence. J Perinat Neonatal Nurs. 2012;26(4):307-16.

16. Basu S, Ratcliffe G. Developing a multidisciplinary approach within the ED towards domestic violence presentations. Emerg Med J. 2014;31(3):192-5.

17. Davis K. Innovative Models of Care Delivery: Addressing Transitions Across The Care Continuum. Chicago: American Hospital Association; 2015.

\section{Endereço para correspondência:}

Ana Paula Reis Carmona

Agrupamento de Centros de Saúde da Amadora

Av. Gorgel do Amaral, 20, $2^{\circ}$ Dto - Águas Livres

939558575 - Amadora - Portugal

E-mail: ana.carmona@arslvt.min-saude.pt 